\title{
Towards Estimating Nadir Objective Vector Using Evolutionary Approaches
}

\author{
Kalyanmoy Deb, Shamik Chaudhuri \\ Kanpur Genetic Algorithms Laboratory \\ Indian Institute of Technology Kanpur \\ Kanpur, PIN 208016, India \\ \{deb,shamik\}@iitk.ac.in
}

\author{
Kaisa Miettinen \\ Helsinki School of Economics \\ PO Box 1210, FI 00101 \\ Helsinki, Finland \\ kaisa.miettinen@hse.fi
}

\begin{abstract}
Nadir point plays an important role in multi-objective optimization because of its importance in estimating the range of objective values corresponding to desired Pareto-optimal solutions and also in using many classical interactive optimization techniques. Since this point corresponds to the worst Pareto-optimal solution of each objective, the task of estimating the nadir point necessitates information about the whole Pareto optimal frontier and is reported to be a difficult task using classical means. In this paper, for the first time, we have proposed a couple of modifications to an existing evolutionary multi-objective optimization procedure to focus its search towards the extreme objective values frontwise. On up to 20-objective optimization problems, both proposed procedures are found to be capable of finding a near nadir point quickly and reliably. Simulation results are interesting and should encourage further studies and applications in estimating the nadir point, a process which should lead to a better interactive procedure of finding and arriving at a desired Pareto-optimal solution.
\end{abstract}

\section{Categories and Subject Descriptors}

G.1 [Numerical Analysis]: Nonlinear programming, unconstrained optimization

\section{General Terms}

Algorithms, performance, experimentation

\section{Keywords}

Nadir objective vector, nadir point, multi-objective optimization, non-dominated sorting GA, evolutionary multiobjective optimization (EMO), ideal point.

\section{INTRODUCTION}

In a multi-objective optimization procedure, an estimation of the nadir objective vector is an important task. The

Permission to make digital or hard copies of all or part of this work for personal or classroom use is granted without fee provided that copies are not made or distributed for profit or commercial advantage and that copies bear this notice and the full citation on the first page. To copy otherwise, to republish, to post on servers or to redistribute to lists, requires prior specific permission and/or a fee.

GECCO'06, July 8-12, 2006, Seattle, Washington, USA

Copyright 2006 ACM 1-59593-186-4/06/0007 ...\$5.00. nadir objective vector represents the worst value of each objective function corresponding to the entire Pareto-optimal set. Sometimes, this point is confused with the point representing the worst objective value of the entire search space, which is often an over-estimation of the nadir objective vector. Along with the ideal objective vector (a point constructed by the best value of each objective), the nadir objective vector is used to normalize objective functions [11], a matter often desired for an adequate functioning of a multiobjective optimization algorithm. With these two extreme values, the objective functions can be scaled so that each scaled objective takes values more or less in the same range. These scaled values can be used for optimization with different algorithms like the weighted-sum approach or the Tchebycheff metric method $[11,3]$. Such a scaling procedure may help in reducing the computational cost by solving the problem faster [13]. Apart from normalizing the objective function values, nadir objective vector is also used for finding Pareto-optimal solutions by different interactive algorithms like guess method and others [11]. The general idea of these methods is to maximize the minimum weighted deviation from the nadir objective vector. Moreover, the knowledge of nadir and ideal objective values helps the decision-maker in adjusting her/his expectations on a realistic level by knowing the range of each objective and can then be used to focus on a desired region. Furthermore, in visualizing Pareto-optimal solutions, the knowledge of the nadir objective vector is essential. Along with the ideal point, the nadir point will then provide the range of each objective to facilitate in visualizing the trade-off information through value paths, bar charts, petal diagrams etc. $[11,12]$.

Since estimating the nadir point necessitates information about the whole Pareto optimal frontier, any procedure of estimating this point should involve finding Pareto-optimal solutions. This makes the task more difficult compared to finding the ideal point [10], which corresponds to the best objective values over the entire search space. Since evolutionary multi-objective optimization (EMO) algorithms attempt to find the entire Pareto-optimal frontier, EMO methodologies stand as viable candidates for this task. However, an estimation of the nadir objective vector need not involve finding intermediate Pareto-optimal solutions. Only extreme solutions corresponding to the Pareto-optimal set will be adequate for the task. In this paper, we have suggested two modifications to an existing EMO methodology - elitist non-dominated sorting GA or NSGA-II [4] - for emphasizing extreme Pareto-optimal solutions, thereby leading to the estimate of the nadir point. Simulation results on 
up to 20-objective (rarely solved using an EMO) optimization problems demonstrate that one of the two approaches - the extremized crowded NSGA-II - is capable of finding a near nadir point more quickly and reliably than the original NSGA-II.

\section{NADIR OBJECTIVE VECTOR}

Multi-objective optimization problems involve a number of conflicting objectives $\left(f_{j}, j=1,2, \ldots, M\right)$ and theoretically give rise to a set of Pareto-optimal solutions, which provide a trade-off among the objectives. Let us consider a two-objective minimization problem, as shown in Figure 1. To calculate the nadir objective vector if $f_{1}$ and $f_{2}$ are maximized individually, we shall obtain points $\mathrm{A}$ and $\mathrm{B}$, respectively. These two points produce an extreme point $\mathbf{z}^{w}$ (termed as the 'worst objective vector' in the figure), which is not the true nadir point. A nadir point must be constructed with the worst objective values of all Paretooptimal solutions, and not with the worst objective values of the entire search space. Thus, to find the nadir point, the Pareto-optimality of the solutions used for constructing the nadir point must be first established. This makes the task of finding the nadir point difficult.

\section{EXISTING METHODS}

To overcome this difficulty, researchers have suggested different methods. Benayoun et al. [1] introduced the first interactive multi-objective optimization method using an estimated nadir point (although authors did not use the term 'nadir'), which was to be found by using a payoff table method. In this method, a table is constructed where $i$-th row of the table represents values of all other objective functions calculated at the point where $i$-th objective is minimum. Thereafter, the maximum value of the $i$-th column can be considered as an estimate of the upper bound of the $i$-th objective and these maximum values together may be used to construct the estimated nadir vector. The main difficulty of such an approach is that corresponding to the minimum solution of an objective there may exist more than one solutions having different values of other objectives, especially in problems having more than two objectives. Consider the Pareto-optimal front of a typical three-objective optimization problem shown in Figure 2. The minimum value of the first objective function is $f_{1}=0$. It can be seen from the figure that there exist a number of solutions having $f_{1}=0$ and different values of $f_{2}$ and $f_{3}$ (all solutions on the line BC). In the payoff table method, when the following three solutions are found $\mathbf{f}^{(1)}=(0,0,1)^{T}$ (point C), $\mathbf{f}^{(2)}=(1,0,0)^{T}$ (point A), and $\mathbf{f}^{(3)}=(0,1,0)^{T}$ (point B) corresponding to minimizations of $f_{1}, f_{2}$, and $f_{3}$, the true nadir point $z^{\text {nad }}=(1,1,1)^{T}$ can be found. However, if solutions $\mathbf{f}^{(1)}=(0,0.2,0.8)^{T}, \mathbf{f}^{(2)}=(0.5,0,0.5)^{T}$ and $\mathbf{f}^{(3)}=(0.7,0.3,0)^{T}$ (marked with open circles) are found corresponding minimizations of $f_{1}, f_{2}$, and $f_{3}$, respectively, a wrong estimate $z^{\prime}=(0.7,0.3,0.8)^{T}$ of the nadir point will be made. The figure shows such a wrong nadir point represents only a portion (shown dark-shaded) of the Paretooptimal front.

Later, Iserman and Steuer [9] demonstrated the difficulties of finding a nadir point even for linear problems and emphasized the need of using a method better than the payoff table method. Dessouky et al. [7] suggested three heuristic methods and Korhonen et al. [10] suggested another heuristic method for this purpose. Let us point out that all these methods suggested have been developed for multi-objective linear problems where all objectives and constraints are linear functions of the variables.

Ehrgott and Tenfelde-Podehl [8] proposed an algorithm based on subproblems, that is, to find the nadir point for an $M$-objective problem, Pareto-optimal solutions of lowerdimensional problems are used. Such a requirement may make the algorithm computationally impractical beyond three objectives. Moreover, authors did not suggest how to extend the idea to nonlinear problems. It must be emphasized that although the determination of the nadir point depends on finding the worst objective values in the set of Paretooptimal solutions, even for linear problems, this is a difficult task.

\section{EVOLUTIONARY APPROACH}

Because the determination of the nadir point is associated with the Pareto-optimal solutions, thereby hinting that a determination of a set of Pareto-optimal solutions will facilitate the estimation of the nadir point. For the past decade or so, evolutionary multi-objective optimization (EMO) algorithms have been gaining popularity because of their ability to find multiple, wide-spread, Pareto-optimal solutions simultaneously in a single simulation run $[3,2]$. Since they aim at finding a set of Pareto-optimal solutions, an EMO procedure can be an ideal way to find the nadir objective vector. However, the naive procedure of first finding a Pareto-optimal set and then determining the nadir objective vector from the set seems to cause a dilemma. Recall that the main purpose of the nadir objective vector is to normalize the objectives so an interactive multi-objective optimization algorithm can be used to find a desired Pareto-optimal solution. However, if an EMO is used to find a representative Pareto-optimal set, there is no major reason for finding the nadir point. In an interactive sense [5], an application of an EMO procedure can provide an idea of the nature of the Pareto-optimal set and subsequent applications of an EMO procedure to a desired (or preferred) region of the Paretooptimal set may be equivalent to the nadir point based interactive methods.

However, EMO procedures use the concept of Paretodominance in their working principles and have been criticized for the ineffectiveness for handling a large number of objectives (more than five objectives or so). To represent a high-dimensional Pareto-optimal front requires an exponentially large number of points [3]. This causes EMO procedures to be inadequate to find the complete Pareto-optimal front in the first place. Thus, for handling a large number of objectives, it may be not advantageous to use the twopronged EMO procedure of finding the Pareto-optimal front and focusing in a desired region. Instead, the EMO procedure may be used to achieve both tasks simultaneously in one application in which the main focus is to distribute the population into those regions of the Pareto-optima set which will construct the nadir point correctly. For the threeobjective minimization problem of Figure 2, the proposed EMO procedure should distribute its population members near regions $\mathrm{A}, \mathrm{B}$, and $\mathrm{C}$, instead of on the entire Paretooptimal set so that the true nadir point can be found quickly. In the following section, we describe two EMO procedures for this purpose. 


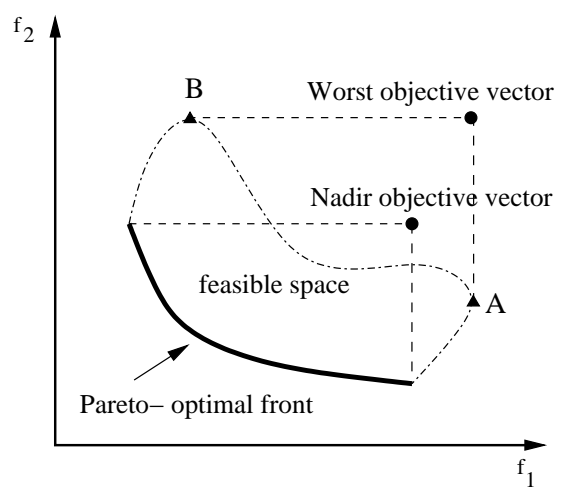

Figure 1: The nadir and worst objective vectors.

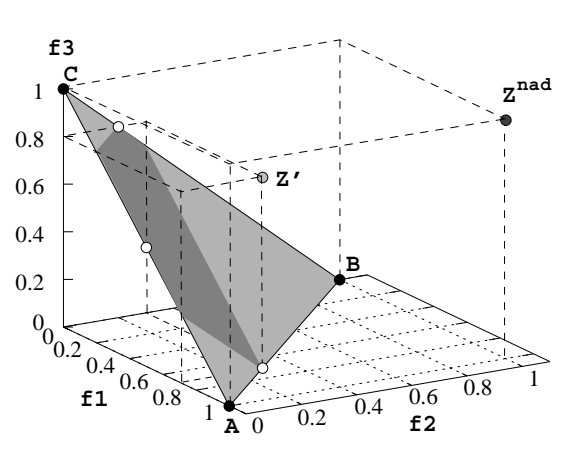

Figure 2: Payoff table method may not find the true nadir point.

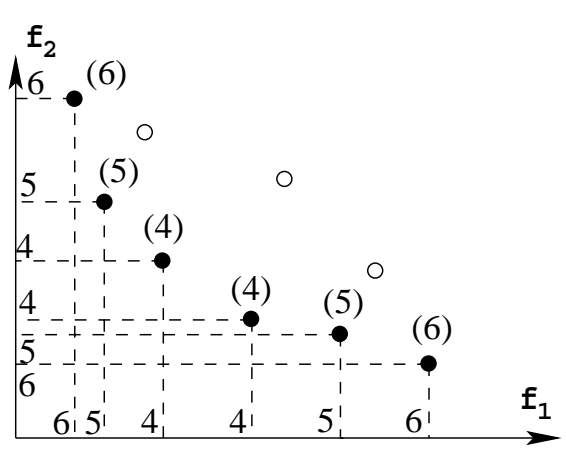

Figure 3: Extremized crowding distance calculation.

\section{ELITIST NON-DOMINATED SORTING GENETIC ALGORITHM (NSGA-II)}

Both EMO procedures proposed here use a specific procedure - elitist non-dominated sorting genetic algorithm (NSGAII) [4] - which has lately received a great deal of attention due to its simplicity, computationally fast approach and availability of source codes.

\subsection{NSGA-II Crowding Distance Calculation}

One primary objective of a multi-objective optimization problem is to preserve diversity along the front. To keep diversity one needs to measure the density of solutions in a non-dominated front. The very simple way of doing it is by clustering using Euclidean distances between solutions, but the amount of computation required is very large. In the original NSGA-II this computation is done in a fast manner. Crowding distance is measured by adding objectivewise normalized difference between two neighboring solutions of a solution $i$. The extreme solutions are assigned an arbitrarily large crowding distance. Thereafter, solutions are sorted from largest crowding distance to lowest and the required number of solutions are picked from the top of the sorted list.

\section{MODIFICATIONS TO NSGA-II}

Though NSGA-II provides importance to the extreme solutions of the non-dominated front, the main emphasis is placed in maintaining a good diversity of solutions on the entire front. However, as discussed above, to find the nadir point we would need to distribute NSGA-II population members around the extreme solutions of the non-dominated front. Thus, for finding the nadir point in a computationally effective manner, we need to emphasize solutions near extreme non-dominated solutions more than that is currently provided by the NSGA-II procedure. To implement such a concept, the crowding distance calculation is modified here.

\subsection{Worst Crowded NSGA-II}

Since the worst objective values for the Pareto-optimal solutions constitute the nadir point, we employ a crowded distance scheme which emphasizes the worst objective values front-wise. Solutions on a particular front are first sorted from minimum to maximum based on each objective (for minimization problems) and a rank equal to the position of the solution in the sorted list is assigned. Thereafter, the maximum rank assigned to a solution due to all objectives is declared as the crowding distance measure. This way, the solution with the maximum objective value of any objective gets the maximum crowded distance. Since the NSGAII procedure emphasizes the non-dominated solutions and solutions having larger crowding distance values, all worst objective values in every front get emphasized, thereby constituting a process which may help find the nadir point.

\subsection{Extremized Crowded NSGA-II}

However, such an emphasis to only the worst objective solutions front-wise may have at least two difficulties: (i) it may not maintain enough diversity in the subpopulation near each worst objective vector, which may cause a premature convergence to a suboptimal solution and (ii) solutions corresponding to the individual worst objective vectors alone may not dominate a number of non-Pareto-optimal solutions, thereby finding a non-dominated front with unwanted solutions. Such a front will then make a wrong estimation of the nadir point. To avoid both these possibilities, we suggest a different crowding distance measure. Solutions on a particular front are first sorted from maximum to minimum based on each objective. A solution closer to either extreme objective vectors (minimum or maximum objective values) gets a higher rank compared to that of an intermediate solution. Thus, the extreme two solutions for every objective get a rank equal to $N^{\prime}$ (number of solutions in the front), the solutions next to these extreme solutions get a rank $\left(N^{\prime}-1\right)$ and so on. Figure 3 shows this rank-assignment procedure. After a rank is assigned to a solution by each objective, the maximum value of the assigned ranks is declared as the crowding distance. The crowding distance values are shown within brackets in the figure. This procedure emphasizes the extreme solutions (minimum and maximum) of each objective and produces a hierarchy by emphasizing the solutions closer to the extreme solutions.

For two-objective problems and for higher-objective problems having a one-dimensional Pareto-optimal front (that is, an $M$-objective problem having $(M-2)$ redundant objectives), this crowding distance assignment is similar to the worst crowding distance assignment scheme (as the minimumrank solution of one objective is the maximum-rank solution of at least one other objective). However, for problems having a large-dimensional Pareto-optimal hyper-surface, the 
effect of extremized crowding is different from that in the worst crowding scheme. In the three-objective problem shown in Figure 2, the extremized crowding scheme will not only emphasize the extreme points $\mathrm{A}, \mathrm{B}$, and $\mathrm{C}$, but also solutions on lines $\mathrm{AB}, \mathrm{BC}$, and $\mathrm{CA}$ and solutions near them. This has two advantages: (i) a good diversity of solutions will be maintained, thereby allowing genetic operators (crossover and mutation) to find better solutions and not causing a premature convergence and (ii) the presence of these solutions will reduce the chance of having spurious non-Paretooptimal solutions to remain in the best non-dominated front, thereby causing a reliable (and stable) computation of the nadir point. Moreover, since the intermediate portion of the Pareto-optimal region is not emphasized, finding the extreme solutions should be quicker than the original NSGAII, especially for problems having a large number of objectives.

\subsection{VEGA Based Approach}

If thought carefully, the worst crowding distance calculation method proposed above has a similarity with another multi-objective GA, known as VEGA (vector evaluated GA) [14]. In this approach, a GA population is subdivided into smaller groups equal to the number of objective functions in each generation. Individuals in the first group are reproduced based on first objective, second group based on second objective, and so on. Although the selection operator of VEGA is restricted to each group, crossover and mutation operators are applied on the entire population as usual. Because of the selective search made for each objective independently to a subpopulation, individuals having better values of each objective are emphasized. Although VEGA has a tendency to converge to the extreme solutions of the Pareto-optimal front, no domination check or no explicit diversity-preserving mechanism (as they are used in NSGA-II) is made in VEGA. In this study, we also evaluate the VEGA approach for estimating the nadir point and compare with the original NSGA-II and with the two proposed modifications of NSGA-II.

\section{TEST PROBLEMS}

The algorithms discussed in Section 6 are tested on a number of test problems starting from two objectives and up to 20 objectives. These test problems are designed in such a way that the complexity level of convergence and shape of the Pareto-optimal front can be tuned by using different parameters and functionals. In these problems, the Paretooptimal front can be determined analytically, thereby allowing to compute the true nadir objective vector. Since the true nadir point is known in these problems, we define the performance measure of an algorithm by simply computing the normalized Euclidean difference $(D)$ between the true nadir point and the obtained nadir point in the objective space.

$$
D=\sqrt{\sum_{i=1}^{M}\left(\frac{z_{i}^{\mathrm{nad}}-z_{i}^{\mathrm{est}}}{z_{i}^{\mathrm{nad}}-z_{i}^{*}}\right)^{2}}
$$

where $z_{i}^{\text {nad }}$ is the true nadir objective value for $i$-th objective function, $z_{i}^{*}$ is the ideal objective value for $i$-th objective vector, and $z_{i}^{\text {est }}$ is the estimated nadir objective value for $i$-th objective vector. The estimated nadir objective vector at any generation is constructed from the solutions of the best non-dominated front of the population at that generation. The above performance metric $D$ is computed at each generation and when a value smaller than a threshold $(\eta=0.01$ used here) is found the simulation is terminated and the algorithm is said to be successful in finding a point close to the true nadir point.

For an application of the proposed approach to any arbitrary problem, first the ideal point $\left(\mathbf{z}^{*}\right)$ and the worst objective vector $\left(\mathbf{z}^{w}\right)$ can be computed by minimizing and maximizing, respectively, each objective function independently. Thereafter, the proposed EMO approach can be applied and the normalized Euclidean distance between the ideal point and the estimated nadir point can be recorded with the generation counter:

$$
\text { Normalized Distance }=\sqrt{\frac{1}{M} \sum_{i=1}^{M}\left(\frac{z_{i}^{\text {est }}-z_{i}^{*}}{z_{i}^{w}-z_{i}^{*}}\right)^{2}} .
$$

If in a problem, the worst objective vector $\mathbf{z}^{w}$ (refer to Figure 1) is the same as the nadir vector, the above normalized distance value will be one. For other scenarios, the normalized distance value will be smaller than one. When the change in this distance value is not significant for a continual number of generations, the algorithm can be terminated and the obtained nadir point can be declared.

To understand the efficiency of each of the proposed algorithms, input parameters are kept fixed for a particular problem. For all problems, we use the SBX crossover with a probability of 0.9 and polynomial mutation operator with a probability of $1 / n$ ( $n$ is the number of variables) and distribution index of 20 [3]. The population size and distribution index for crossover are set according to the problem and are mentioned below. Each algorithm is run 11 times, each time starting from a different random initial population, however all algorithms are started with identical initial populations. The number of generations required to satisfy the termination criterion $(D \leq \eta)$ is noted for each simulation run and the best, median and worst number of generations are presented.

\subsection{Two-Objective Problems}

Actually, the payoff table method can be reliably used to find the nadir point for a two-objective optimization problem and there is no need to use an evolutionary approach. It is the higher-objective problems in which the payoff table and other suggested methods have difficulties and there is a strong need for other better methods. However, here we still apply the proposed EMO procedures to a few two-objective problems for completeness.

We have chosen five test problems (ZDT1 to ZDT4 and ZDT6) for performance measure of the proposed algorithms. Nadir objective vectors for ZDT1, ZDT2 and ZDT4 are $(1,1)^{T}$ and that for ZDT3 and ZDT6 are $(0.85,1.0)^{T}$ and $(1,0.92)^{T}$, respectively. Among them ZDT4 is the most difficult due to the presence of 99 local non-dominated fronts.

Table 1 shows the number of generations needed to find a near nadir point (within $\eta=0.01$ ) by different algorithms. For comparatively easier problems (ZDT1, ZDT2 and ZDT3), we use a crossover index of 2 and for ZDT4 and ZDT6, we use a value of 10. It is clear from the results that the performances of the worst crowded and extremized crowded NSGA-II are more or less the same and are slightly 
Table 1: Comparative results for two-objective problems.

\begin{tabular}{|c|c|c|c|c|c|c|c|c|c|c|}
\hline \multirow{3}{*}{$\begin{array}{c}\text { Test } \\
\text { Problem }\end{array}$} & \multirow{3}{*}{$\begin{array}{l}\text { Pop. } \\
\text { size }\end{array}$} & \multicolumn{9}{|c|}{ Number of generations to reach $D \leq 0.01$} \\
\hline & & \multicolumn{3}{|c|}{ NSGA-II } & \multicolumn{3}{|c|}{ Worst crowd. NSGA-II } & \multicolumn{3}{|c|}{ Extr. crowd. NSGA-II } \\
\hline & & Best & Median & $\overline{\text { Worst }}$ & Best & Median & Worst & Best & Median & Worst \\
\hline ZDT1 & 100 & 32 & 44 & 80 & 31 & 41 & 45 & 31 & 42 & 48 \\
\hline ZDT2 & 100 & 45 & 56 & 83 & $\overline{44}$ & 72 & 97 & 34 & 67 & 93 \\
\hline ZDT3 & 100 & 33 & 40 & 55 & 33 & 40 & 113 & 28 & 36 & 45 \\
\hline ZDT4 & 100 & 176 & 197 & 257 & 148 & 201 & 224 & 165 & 191 & 219 \\
\hline ZDT6 & 100 & 137 & 151 & 161 & 125 & 130 & 143 & 126 & 132 & 135 \\
\hline
\end{tabular}

better than the original NSGA-II algorithm for more complex problems, such as ZDT4 and ZDT6.

It is concluded that for two-objective optimization problems the use of extremized crowding or the worst crowded approach may not be necessary. The procedure of finding the complete Pareto-optimal front and then deriving the nadir point from the front is a computationally viable approach for two-objective problems, but as discussed earlier, may be replaced by the conventional pay-off table method.

Before we present the results for problems having many objectives, let us investigate how the VEGA approach would fair in estimating the nadir point in the ZDT test problems. We use VEGA with 500 population members and run it for 1,000 generations. Even with such a large number of evaluations, the VEGA approach could not find the nadir objective vector, as shown in Table 2 . The table shows that there is not much of a reduction in the normalized Euclidean difference (equation 1) of the estimated nadir point from the actual one from the initial to the final generation. Because of it's inability to solve these two-objective problems (in which the NSGA-II approach and its modifications performed well), the VEGA approach is not applied for higherobjective problems.

Table 2: Performance of the VEGA approach in estimating the nadir point on ZDT problems.

\begin{tabular}{|c|r|r|r|}
\hline Test & Pop. & \multicolumn{2}{|c|}{ Difference $(D)$ from true nadir point } \\
\cline { 3 - 4 } problem & size & Initial pop. & \multicolumn{1}{|c|}{ Final pop. } \\
\hline ZDT1 & 500 & 12.36 & 7.77 \\
\hline ZDT2 & 500 & 6.55 & 3.48 \\
\hline ZDT3 & 500 & 3.17 & 3.28 \\
\hline ZDT4 & 500 & 145.82 & 149.45 \\
\hline ZDT6 & 500 & 9.06 & 9.34 \\
\hline
\end{tabular}

\subsection{Problems with More Objectives}

To test the algorithms for three and more objectives, we choose three DTLZ test problems [6]. These problems are designed in a manner so that they can be extended to any number of objectives. Nadir objective vectors of $M$-objective DTLZ1, DTLZ2 and DTLZ5 are $(0.5, \ldots, 0.5)^{T},(1, \ldots, 1)^{T}$ and $\left(\left(\frac{1}{\sqrt{2}}\right)^{M-2},\left(\frac{1}{\sqrt{2}}\right)^{M-2},\left(\frac{1}{\sqrt{2}}\right)^{M-3},\left(\frac{1}{\sqrt{2}}\right)^{M-4}, \ldots,\left(\frac{1}{\sqrt{2}}\right)^{0}\right)^{T}$ respectively. The variable mapping of third test problem, DTLZ5, is somewhat modified from the original DTLZ5:

$$
\theta_{i}= \begin{cases}\frac{\pi}{2} x_{i}, & \text { for } i=1, \\ \frac{\pi}{4\left(1+g^{\prime}\right)}\left(1+2 g^{\prime} x_{i}\right), & \text { for } i \in I=\{2,3, \ldots, M-1\},\end{cases}
$$

$$
\begin{aligned}
& g\left(\mathbf{x}_{M}\right)=\sum_{x_{i} \in \mathbf{x}_{M}} 100\left(x_{i}-0.5\right)^{2}, \quad g^{\prime}=\min \left(0.3, g\left(\mathbf{x}_{M}\right)\right) \\
& L \leq x_{i} \leq U \quad \text { for } i \in I, \quad 0 \leq x_{i} \leq 1 \quad \text { for } i \notin I
\end{aligned}
$$

\subsubsection{Three-Objective DTLZ Problems}

All three algorithms are run with 100 population members for three-objective DTLZ1, DTLZ2 and DTLZ5. For DTLZ5, we use bounds $L=0.1$ and $U=0.9$. Table 3 shows the number of generations needed to find a solution close to (within a normalized difference of 0.01 ) the actual nadir point. It is observed that the worst crowded NSGA-II and the extremized crowded NSGA-II perform more or less similar to each other and are somewhat better than the original NSGA-II.

\subsubsection{Five-Objective DTLZ Problems}

Next, we study the performance of all three NSGA-II procedures on five-objective DTLZ problems. It is now quite evident from Table 4 that the proposed modifications to the NSGA-II procedure perform much better than the original NSGA-II. The best simulation of the original NSGA-II takes 2,342 generations to estimate the nadir point, whereas the extremized crowded NSGA-II requires only 353 generations and the worst-crowded NSGA-II requires 611 generations for the DTLZ1 problem. In the case of DTLZ2 problem, the minimum number of generations required for the original NSGA-II, extremized crowded NSGA-II and the worst crowded NSGA-II are 650, 94 and 139, respectively. The median generation counts of the modified NSGA-II methods for 11 independent runs are also much better than that of the original NSGA-II. Since the original NSGA-II procedure attempts to find the entire five-dimensional Pareto-optimal set properly before a true estimate of the nadir point can be made, it requires more generations for problems with a large number of objectives.

The difference between the worst crowded and extremized crowded NSGA-II methods is also clear from the table. For a problem having a large number of objectives, the extremized crowded NSGA-II emphasizes both best and worst extreme solutions for each objective maintaining an adequate diversity among the population members. The NSGA-II operators are able to exploit such a diverse population and make a faster progress towards the extreme Pareto-optimal solutions needed to estimate the nadir point correctly. These results imply that for a problem having a large number of objectives, an emphasis for the individual-best solutions (instead of all non-dominated solutions) is a faster approach for locating the nadir point. Figure 4 shows the convergence rate for the best runs of three algorithms on DTLZ1.

For the DTLZ5 problem, we change the bounds to $L=$ 0.4 and $U=0.6$. Simulation results show that there is no significant advantage of the proposed modifications, be- 
Table 3: Comparative results for three-objective DTLZ problems.

\begin{tabular}{|c|c|c|c|c|c|c|c|c|c|c|}
\hline \multirow{3}{*}{$\begin{array}{c}\text { Test } \\
\text { problem }\end{array}$} & \multirow{3}{*}{$\begin{array}{l}\text { Pop. } \\
\text { size }\end{array}$} & \multicolumn{9}{|c|}{ Number of generations to reach $D \leq 0.01$} \\
\hline & & \multicolumn{3}{|c|}{ NSGA-II } & \multicolumn{3}{|c|}{ Worst crowd. NSGA-II } & \multicolumn{3}{|c|}{ Extr. crowd. NSGA-II } \\
\hline & & Best & Median & Worst & Best & Median & Worst & Best & Median & Worst \\
\hline DTLZ1 & 100 & 223 & 366 & 610 & 171 & 282 & 345 & 188 & 265 & 457 \\
\hline DTLZ2 & 100 & 75 & 111 & 151 & 38 & 47 & 54 & 41 & 49 & 55 \\
\hline DTLZ5 & 100 & 63 & 80 & 104 & 59 & 74 & 86 & 62 & 73 & 88 \\
\hline
\end{tabular}

Table 4: Comparative results for five-objective DTLZ problems.

\begin{tabular}{|c|c||c|c|c||c|c|c||c|c|c|}
\hline \multirow{2}{*}{$\begin{array}{c}\text { Test } \\
\text { problem }\end{array}$} & \multirow{2}{*}{$\begin{array}{l}\text { Pop. } \\
\text { size }\end{array}$} & \multicolumn{7}{|c|}{ Number of generations to reach $D \leq 0.01$} \\
\cline { 3 - 10 } & & \multicolumn{3}{|c|}{ NSGA-II } & Worst crowd. NSGA-II & \multicolumn{1}{|c|}{ Extr. crowded NSGA-II } \\
\cline { 3 - 10 } & & Best & Median & Worst & Best & Median & Worst & Best & Median & Worst \\
\hline DTLZ1 & 100 & 2,342 & 3,136 & 3,714 & 611 & 790 & 1,027 & 353 & 584 & 1,071 \\
\hline DTLZ2 & 100 & 650 & 2,142 & 5,937 & 139 & 166 & 185 & 94 & 114 & 142 \\
\hline DTLZ5 & 100 & 52 & 66 & 77 & 51 & 66 & 76 & 49 & 61 & 73 \\
\hline
\end{tabular}

Table 5: Comparative results for 10-objective DTLZ problems.

\begin{tabular}{|c|c|c|c|c|c|c|c|c|c|c|}
\hline \multirow{2}{*}{$\begin{array}{c}\text { Test } \\
\text { problem }\end{array}$} & \multirow{2}{*}{$\begin{array}{l}\text { Pop } \\
\text { size }\end{array}$} & \multicolumn{9}{|c|}{ Number of generations to reach $D \leq 0.01$} \\
\hline & & \multicolumn{3}{|c|}{ NSGA-II } & \multicolumn{3}{|c|}{ Worst crowd. NSGA-II } & \multicolumn{3}{|c|}{ Extr. crowd. NSGA-II } \\
\hline & & Best & Median & Worst & Best & Median & Worst & Best & Median & Worst \\
\hline DTLZ1 & 200 & 17,581 & 21,484 & 33,977 & 1,403 & 1,760 & 2,540 & $\overline{1,199}$ & 1,371 & 1,790 \\
\hline DTLZ2 & 200 & - & - & - & 520 & 823 & 1,456 & 388 & 464 & 640 \\
\hline DTLZ5 & 200 & 45 & 53 & 60 & 43 & 53 & 57 & 45 & 51 & 64 \\
\hline
\end{tabular}

cause the five-objective problem degenerates to have a onedimensional curve as the Pareto-optimal front and all methods find it easier to locate the true nadir point quickly.

\subsubsection{Ten-Objective DTLZ Problems}

Table 5 presents the number of generations required to find a point close (within $\eta=0.01$ ) to the nadir point by the three NSGA-II procedures for 10-objective DTLZ problems. It is clear that the extremized crowded NSGA-II performs an order of magnitude better than the original NSGA-II and is also better than the worst crowded NSGA-II. Both DTLZ1 and DTLZ2 problems have 10-dimensional Paretooptimal fronts and the extremized crowded NSGA-II makes a good balance of maintaining diversity and emphasizing worst objective vectors so as to make the nadir point estimation quick. In the case of 10-objective DTLZ2 problem, the original NSGA-II could not even find the nadir objective vector after 50,000 generations (and achieved a normalized difference measure (equation 1) of 5.936). Figure 5 shows a typical convergence pattern of the proposed and original NSGA-II algorithms on 10-objective DTLZ1. The plots demonstrate that for a large number of generations the estimated nadir point is away from the true nadir point, but after some generations (around 1,000 in this problem) the estimated nadir point comes quickly near the true point. To understand the dynamics of the movement of the extremized crowded NSGA-II population with generation counter, we have counted the number of solutions in the population which dominate the true nadir point and plotted in Figures 5. In DTLZ1, it is seen that the first point dominating the actual nadir point appears in the population at around 750 generations and since then when an adequate number of such solutions appear in the population, the population very quickly converges to the correct Pareto-optimal regions for estimating the nadir point. A similar phenomenon occurs for the worst crowded NSGA-II, but is not shown here for brevity. The figure also plots the number of non-dominated points for the original NSGA-II and a similar dynamics is observed, with a much delayed convergence.

In case of DTLZ5 problem, we change the bounds to $L=$ 0.45 and $U=0.55$. The performance of all three algorithms are almost the same, due to the reasons outlined in previous subsection.

\subsubsection{5-Objective DTLZ Problems}

Next, we apply all three NSGA-II procedures on 15-objective DTLZ1 and DTLZ2 problems. Table 6 shows number of generations needed to find a point within a normalized Euclidean difference of 0.01 from the true nadir point with 500 population members. The original NSGA-II simulations were run for a maximum of 50,000 generations and the best normalized Euclidean difference measure (equation 1) comes at 3554.587 and 8.632 (which is much larger than zero) for DTLZ1 and DTLZ2, respectively, whereas both proposed modifications of NSGA-II are able to find a good estimate of the nadir point in a reasonable number of evaluations for both problems.

\subsection{Scale-up Performance}

We now compare the overall function evaluations needed to estimate the nadir point within a normalized difference measure (equation 1) of 0.01 from the true nadir point for DTLZ2 test problem by the extremized crowded NSGA-II. Although we presented the results for problems up to 15 


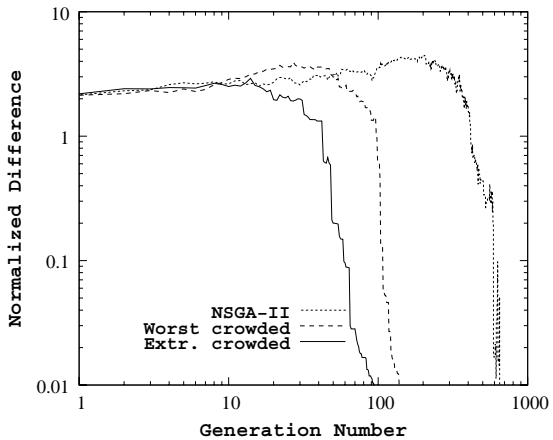

Figure 4: Performance of three methods on five-objective DTLZ2.

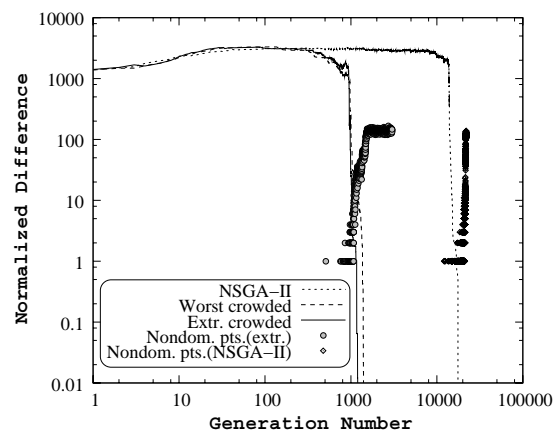

Figure 5: Performance of three methods on 10-objective DTLZ1.

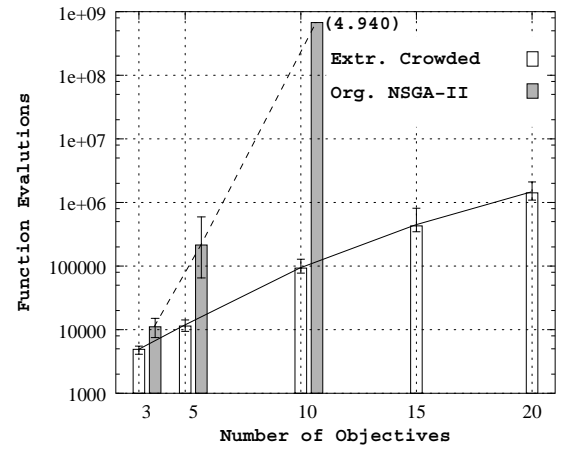

Figure 6: Function evaluations versus number of objectives for DTLZ2.
Table 6: Results for 15-objective DTLZ problems.

\begin{tabular}{|c||c|c|c||c|c|c|}
\hline \multicolumn{1}{|c||}{} & \multicolumn{1}{c||}{$\begin{array}{c}\text { Prob- } \\
\text { lem }\end{array}$} & \multicolumn{5}{c|}{ Number of generations for $D \leq 0.01$} \\
\cline { 2 - 7 } & Worst cwd. NSGA-II & Extr. crd. NSGA-II \\
\cline { 2 - 7 } & Best & Med. & Worst & Best & Med. & Worst \\
\hline DTLZ1 & 2,567 & 3,144 & 8,645 & 1,829 & 2,372 & 2,841 \\
\hline DTLZ2 & 988 & 1,166 & 1,729 & 693 & 858 & 1,620 \\
\hline
\end{tabular}

objectives in earlier subsections, here we show scale-up performance of the extremized crowded NSGA-II up to 20 objectives. Figure 6 shows the performance on DTLZ2. After 670 million function evaluations, the original NSGA-II is still not able to come close (reaches a point with a normalized difference of 4.940) to the true nadir point. However, the extremized crowded NSGA-II takes an average only 429,000 evaluations to reach a point very close (with a normalized difference of 0.01) to the true nadir point. Because of the computational inefficiencies associated with the original NSGA-II approach, we do not perform any simulation for 15 or more objective DTLZ2 problem.

The nature of the plots for the extremized crowded NSGAII is found to be sub-linear on logarithmic axes. This indicates that a lesser than exponential scaling property of the proposed extremized crowded NSGA-II. It is important to highlight here that estimating the nadir point requires identification of the worst objective vector corresponding to the Pareto-optimal solutions. Since this requires that an evolutionary procedure first puts its population members on the Pareto-optimal front, an adequate computational effort must be spent to achieve this task. However, the earlier tables have indicated that the computational effort needed with the extremized crowded NSGA-II is much smaller compared to the original NSGA-II, which attempts to find the entire Pareto-optimal front and then constructs the nadir point.

\section{A 3-OBJECTIVE LINEAR PROBLEM}

The following linear problem was found to be difficult for estimating the nadir point [10]:

$$
\text { Maximize } 11 x_{2}+11 x_{3}+12 x_{4}+9 x_{5}+9 x_{6}-9 x_{7},
$$

$$
\begin{aligned}
\text { Maximize } & 11 x_{1}+11 x_{3}+9 x_{4}+12 x_{5}+9 x_{6}-9 x_{7}, \\
\text { Maximize } & 11 x_{1}+11 x_{2}+9 x_{4}+9 x_{5}+12 x_{6}+12 x_{7}, \\
\text { Subject to } & \sum_{i=1}^{7} x_{i}=1, \quad x_{i} \geq 0, \quad i=1,2, \ldots, 7
\end{aligned}
$$

To use an evolutionary optimization procedure, we bound $x_{i} \in[0,1]$ and perform the following repair mechanism: $x_{i} \leftarrow x_{i} / \sum_{i=1}^{7} x_{i}$ to make every solution a feasible one. All three NSGA-II approaches are applied with a standard parameter setting: population size $=100$, crossover probability $=0.9$, crossover index $=10$, mutation probability $=$ $1 / 7$, and mutation index $=20$. Figure 7 shows the populations obtained with NSGA-II and the extremized crowded NSGA-II approaches. The nadir point obtained using both

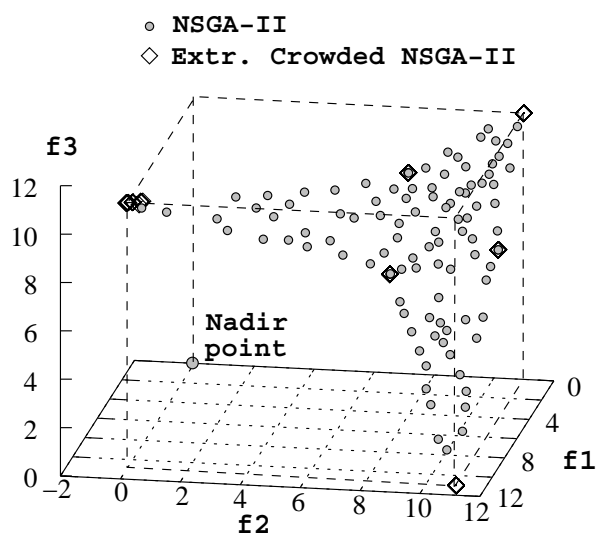

Figure 7: Distribution of points on linear problem.

approaches is $(0,0,0)^{T}$. It is interesting to note how the extremized crowded NSGA-II is able to find both minimum and maximum objective values corresponding to the Paretooptimal solutions, the presence of which allows a correct estimation of the nadir point.

In Figure 8, we plot the normalized distance metric (equation 2) versus generation number. In this problem, the ideal vector is $\mathbf{z}^{*}=(12,12,12)^{T}$ and the worst objective vector is $\mathbf{z}^{w}=(-9,-9,0)^{T}$ (computed using a single-objective EA). The true nadir point, the normalized distance measure is 0.7423 . The figure shows that the extremized crowded NSGA-II steadily reaches this value, whereas the worst crowded NSGA-II is unable to estimate the nadir vector reliably. In 
the worst crowded NSGA-II, since only worst solutions are emphasized, a frontier with these solutions allow a number of non-Pareto-optimal solutions to coexist. The presence of these spurious solutions makes a wrong estimate of the nadir point. However, the presence of individual best solutions (as shown in Figure 7), in addition to the worst Pareto-optimal solutions, reduce the chance of having spurious solutions, thereby causing a stable and correct estimation of the nadir point.

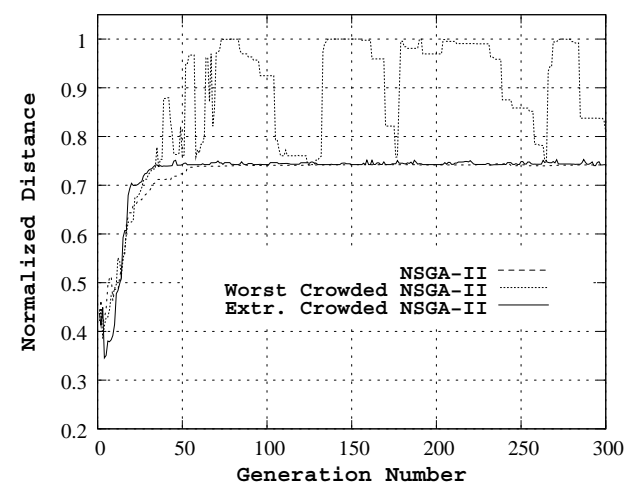

Figure 8: A quick and stable convergence of extremized crowded NSGA-II.

\section{CONCLUSIONS}

In this paper, we have proposed a couple of modifications to the NSGA-II procedure for estimating the nadir point in a multi-objective optimization problem. By definition, a nadir point is constructed with the worst objective values corresponding to the Pareto-optimal solutions. Since this requires finding the Pareto-optimal solutions, it is a difficult task. Both the proposed methodologies emphasize the extreme solutions corresponding to each objective front-wise. Since intermediate Pareto-optimal solutions are not important in this task, both procedures have been found to be capable of making a quicker estimate of the nadir point than the original NSGA-II procedure for a number of test problems having two to 20 objectives (rarely used in EMO literature). Based on the study, we conclude the following: (i) Emphasizing both best and worst objective values front-wise has been found to be a better approach than emphasizing only the worst objective values front-wise for solving largedimensional problems. Since the former approach maintains a diverse set of solutions near worst front-wise objective values, the search is better. (ii) The computational effort to estimate the nadir point has been observed to be much better (more than an order of magnitude) for many objectives than the original NSGA-II procedure. (iii) For a fewer objectives (up to three-objective) and DTLZ5 problems with a lower-dimensional Pareto-optimal front, both proposed approaches have been observed to perform well. The performance of original NSGA-II has also been found to be similar to the proposed approaches. Thus, for problems having small number of objectives (say up to three-objective or so) and problems having a low-dimensional Pareto-optimal front, there is no motivation to find the nadir point explicitly using the novel evolutionary approaches. The use of NSGA-II or an equivalent method may be adequate to find a well-represented set of Pareto-optimal solutions, which can then be used for a decision-making. However, if the decisionmaker prefers to use an interactive method, the proposed methodologies can also be used to estimate the nadir point for the purpose. On the other hand, for many objectives, the nadir point may be estimated quickly and reliably using the proposed extremized crowded NSGA-II. Thereafter, a classical procedure (using both ideal and nadir points) can be applied interactively with a decision-maker to find a desired Pareto-optimal solution.

\section{Acknowledgments}

Authors acknowledge the support provided by STMicroelectronics, Agarte and Noida for performing this study.

\section{REFERENCES}

[1] R. Benayoun, J. de Montgolfier, J. Tergny, and P. Laritchev. Linear programming with multiple objective functions: Step method (STEM). Mathematical Programming, 1(3):366-375, 1971.

[2] C. A. C. Coello, D. A. VanVeldhuizen, and G. Lamont. Evolutionary Algorithms for Solving Multi-Objective Problems. Boston, MA: Kluwer Academic Publishers, 2002.

[3] K. Deb. Multi-objective optimization using evolutionary algorithms. Chichester, UK: Wiley, 2001.

[4] K. Deb, S. Agrawal, A. Pratap, and T. Meyarivan. A fast and elitist multi-objective genetic algorithm: NSGA-II. IEEE Transactions on Evolutionary Computation, 6(2):182-197, 2002.

[5] K. Deb and S. Chaudhuri. I-EMO: An interactive evolutionary multi-objective optimization tool. Technical Report KanGAL Report Number 2005005, Department of Mechanical Engineering, IIT Kanpur, India, 2005. Also in Proceedings of Pattern Recognition in Machine Intelligence (PReMI'05), Springer.

[6] K. Deb, L. Thiele, M. Laumanns, and E. Zitzler. Scalable test problems for evolutionary multi-objective optimization. In A. Abraham, L. Jain, and R. Goldberg, editors, Evolutionary Multiobjective Optimization, pages 105-145. London: Springer-Verlag, 2005.

[7] M. I. Dessouky, M. Ghiassi, and W. J. Davis. Estimates of the minimum nondominated criterion values in multiple-criteria decision-making. Engineering Costs and Production Economics, 10:95-104, 1986.

[8] M. Ehrgott and D. Tenfelde-Podehl. Computation of ideal and nadir values and implications for their use in MCDM methods. European Journal of Operational Research, 151:119-139, 2003.

[9] H. Iserman and R. E. Steuer. Computational experience concerning payoff tables and minimum criterion values over the efficient set. European Journal of Operational Research, 33(1):91-97, 1988.

[10] P. Korhonen, S. Salo, and R. Steuer. A heuristic for estimating nadir criterion values in multiple objective linear programming. Operations Research, 45(5):751-757, 1997.

[11] K. Miettinen. Nonlinear Multiobjective Optimization. Kluwer, Boston, 1999.

[12] K. Miettinen. Graphical illustration of Pareto optimal solutions. In T. Tanino, T. Tanaka, and M. Inuiguchi, editors, Multi-Objective Programming and Goal Programming: Theory and Applications, pages 197-202. Springer-Verlag, Berlin, Heidelberg, 2003.

[13] K. Miettinen, M. M. Mäkelä, and K. Kaario. Experiments with classification-based scalarizing functions in interactive multiobjective optimization.

[14] J. D. Schaffer. Multiple objective optimization with vector evaluated genetic algorithms. In Proceedings of the First International Conference on Genetic Algorithms, pages 93-100, 1985. 